\title{
THE GLOBULAR CLUSTER CONTENT OF MAFFEI 1
}

\author{
T. J. Davidge ${ }^{1}$ \\ Canadian Gemini Office, Herzberg Institute of Astrophysics, \\ National Research Council of Canada, 5071 West Saanich Road, \\ Victoria, B.C. Canada V9E 2E7 \\ email: tim.davidge@nrc.ca \\ Sidney van den Bergh \\ Herzberg Institute of Astrophysics, \\ National Research Council of Canada, 5071 West Saanich Road, \\ Victoria, B.C. Canada V9E 2E7 \\ email: sidney.vandenbergh@nrc.ca
}

\begin{abstract}
Near-infrared images have been used to search for bright globular clusters near the center of the elliptical galaxy Maffei 1. The number of objects in various radial intervals from the center of Maffei 1 are compared with the number density of sources in two control fields, and we find an excess of $31 \pm 11$ objects with $K$ between 16.6 and 18.0 in an annulus between 20 and $90 \operatorname{arcsec}$ from the galaxy center. At radii in excess of 90 arcsec the ability to find clusters is frustrated by statistical noise in the foreground star population. It is demonstrated that the globular clusters located within 90 arcsec of the center of Maffei 1 span a range of near-infrared colors that are at least as great as that which is seen among M31 clusters; some of the clusters appear to have colors that are consistent with them being very young. The specific globular cluster frequency within 90 arcsec of the center of Maffei 1 is $\mathrm{S}_{N}=1.3 \pm 0.6$ if the distance modulus is $28.1 \pm 0.2$ and $\mathrm{A}_{V}=4.7 \pm 0.2$. The specific cluster frequency of Maffei 1 is thus similar to that of other nearby field elliptical galaxies.
\end{abstract}

Subject headings: galaxies: individual (Maffei 1) - galaxies: elliptical and lenticular, cD - galaxies: star clusters

\footnotetext{
${ }^{1}$ Visiting Astronomer, Canada-France-Hawaii Telescope, which is operated by the National Research Council of Canada, the Centre National de le Recherche Scientifique, and the University of Hawaii.
} 


\section{INTRODUCTION}

With typical masses near $10^{5} \mathrm{M}_{\odot}$, globular clusters likely formed in molecular complexes that are much larger than those in the solar neighborhood, where the vast majority of molecular clouds have $\log \left(\mathrm{M}_{\odot}\right)<5-6$ (e.g. Solomon \& Rivolo 1989). As noted by Harris \& Pudritz (1994), the conditions required for the supergiant molecular clouds (SGMCs) that are likely globular cluster nurseries to form in large numbers are associated with significant events in the evolution of a galaxy, such as the initial collapse of protogalactic fragments and major mergers, both of which spur the cloud-cloud collisions that are thought to be needed for the assembly of large star-forming complexes. Events of this nature can occur at various times in the life of a galaxy; consequently, the clusters in a galaxy may not be coeval, but may have formed during different times and under different conditions. Indeed, many elliptical galaxies have red and blue globular clusters, and the absence of a correlation between the metallicity of blue globular clusters and host galaxy properties (e.g. Forbes \& Forte 2001, but see also Strader, Brodie, \& Forbes 2004) suggests that these objects formed very early on, possibly in autonomous mini-halos (e.g. Beasley et al. 2003). On the other hand, there are indications that red globular clusters may have formed after the potential well of the final galaxy had been defined, possibly during mergers (e.g. Kundu \& Whitmore 2001). Some objects that appear to be globular clusters at the present day may actually be the cores of disrupted satellites (e.g. van den Bergh \& Mackey 2004; Bekki \& Freeman 2003; Mizutani, Chiba, \& Sakamoto 2003; Meylan et al. 2001).

The events that lead to the formation of SGMCs are believed to be associated with the creation of pressure-supported, as opposed to rotationally supported, structures. It might thus be anticipated that the specific frequency of globular clusters, $\mathrm{S}_{N}$ (Harris \& van den Bergh 1981), should be higher in galaxies dominated by spheroids, rather than disks. This expectation is broadly consistent with observations, although there also appears to be an environmental dependence, in the sense that $S_{N}$ tends to be highest among elliptical galaxies near the centers of rich clusters (e.g. Harris 1991, but see also Kundu \& Whitmore 2001). While it has been suggested that some Galactic globular clusters may be associated with the disk (e.g. Zinn 1985), these more likely formed with the bulge (e.g. Minniti 1995).

Studies of the globular clusters systems around nearby galaxies provide an obvious means of testing our understanding of the formation and evolution of globular clusters. As one of the nearest elliptical galaxies, Maffei 1 is a potentially important target for a survey of globular cluster content. A complicating factor is that Maffei 1 is viewed through the Galactic disk, and so there is significant obscuration by dust and contamination from foreground stars. The extinction towards Maffei 1 is patchy, and individual dense dust clouds are seen projected against the central regions of the galaxy (e.g. Buta \& McCall 
2003).

Maffei 1 might be expected to have a large number of clusters. Indeed, elliptical galaxies have a mean specific cluster frequency $\mathrm{S}_{N}=2.4 \pm 0.8$ (Kundu \& Whitmore 2001). Therefore, with $V=11.1$ (Buta \& McCall 1999), so that $\mathrm{M}_{V}=-21.7$, Maffei 1 should have an entourage of $\sim 1150$ clusters if it is typical of other elliptical galaxies. However, only a modest number of clusters have been detected to date. Davidge (2002) used $J, H$, and $K^{\prime}$ images obtained with the CFHT AOB + KIR imager to search for globular clusters in the central $700 \times 700$ parsec $^{2}$ of this galaxy, and no obvious cluster candidates brighter than the peak of the globular cluster luminosity function (GCLF) were detected. Using WFPC2 images, Buta \& McCall (2003) identified 20 non-stellar objects that have sizes and brightnesses which are consistent with them being clusters. However, the mean $R-I$ colors of these objects, as well as the scatter about the mean, differ from those of the Galactic globular cluster system. It is not clear if these differences in photometric properties are intrinsic to clusters in Maffei 1, or if they are a consequence of differential reddening.

In the present study, broad-band near-infrared images are used to search for globular clusters in the central $6 \times 6 \operatorname{arcmin}^{2}\left(7.3 \times 7.3 \mathrm{kpc}^{2}\right)$ of Maffei 1 . This area contains slightly more than half of the total light from Maffei 1 (Buta \& McCall 1999), and so should also contain a significant fraction of the Maffei 1 cluster system. A search for globular clusters in Maffei 1 at near-infrared wavelengths has two advantages when compared with searches at visible wavelengths. First, the extinction is much smaller in the infrared, with $\mathrm{A}_{K} / \mathrm{A}_{V} \sim 0.11$ (Rieke \& Lebofsky 1985). Second, the broad-band near-infrared spectral-energy distributions (SEDs) of stars are sensitive to surface gravity, thereby simplifying the task of distinguishing between the low surface gravity giants that dominate the integrated spectra of globular clusters and the high surface gravity main sequence stars that occur in large numbers in the foreground disk. This behaviour is clearly seen in the $(J-H, H-K)$ two-color diagram (TCD), which is used to investigate the SEDs of sources towards Maffei 1 in this paper.

Reddening and distance estimates for Maffei 1 are summarized in Tables 1 and 2. For the current study we select the median value in each table, and so a distance modulus of $28.1 \pm 0.2$ and a foreground absorption $\mathrm{A}_{V}=4.7 \pm 0.2$ magnitudes are adopted throughout the paper.

The paper is structured as follows. Details of the observations and the data reduction procedures are reported in $\S 2$, while the photometric measurements and the results of the search for globular clusters are presented in $\S 3$. The $S_{N}$ of the central regions of Maffei 1 is estimated in $\S 4$, while a discussion and summary of the results follows in $\S 5$. 


\section{OBSERVATIONS \& REDUCTIONS}

The data were recorded during the nights of November 21 and 22 (UT) 2002 with the CFHTIR imager, which was mounted at the Cassegrain focus of the 3.6 metre Canada-France-Hawaii Telescope (CFHT). The detector in the CFHTIR is a $1024 \times 1024$ $\mathrm{HgCdTe}$ array. Each pixel subtends 0.22 arcsec on a side, and so individual exposures cover a $3.8 \times 3.8 \operatorname{arcmin}^{2}$ area.

The central $6 \times 6 \operatorname{arcmin}^{2}$ of Maffei 1 was imaged by recording exposures at four different pointings. Images were recorded through $J, H$, and $K^{\prime}$ filters with a total exposure time of 12 minutes per filter per pointing. The layout of the pointings on the sky is shown in Figure 1. Also shown in Figure 1 is the $\mu_{I}=22.77$ magnitude $\operatorname{arcsec}^{-2}$ isophote, which encompasses half of the total light from Maffei 1 and was computed using the structural information plotted in Figures 23 and 24 of Buta \& McCall (1999). Adjacent pointings overlap, and sources in the regions that are common to adjacent pointings were used to assess photometric stability $(\S 3.1)$.

Contamination by foreground stars is a major consideration when observing Maffei 1. Two control fields, located 30 arcminutes to the north and south of the galaxy center, were observed with the same filters and exposure times as Maffei 1 to monitor foreground star contamination. The co-ordinates of the control fields are listed in Table 3.

Calibration images were acquired at various times during the three night observing run. Dome flats were recorded at the beginning of each night, while the detector dark current was measured at the end of each night. Neither the dark current nor flat field pattern showed night-to-night variations, and so the relevant data from individual nights

were combined to construct master dark and flat-field frames for the entire run. Regions of the sky with low stellar surface density were also observed periodically, and these were used to construct calibration images that monitor interference fringes and thermal emission from warm objects along the optical path.

The data were processed using a standard sequence for infrared imaging that removes signatures introduced by the telescope, the instrumentation, and the atmosphere. The basic steps in this sequence are (1) the subtraction of a dark frame, (2) the division by a flat-field frame, (3) the subtraction of the DC sky level from each exposure, and (4) the subtraction of a fringe/thermal emission calibration frame. The processed images for each field + filter combination were spatially registered to correct for dither offsets made during acquisition and then median combined. The resulting combined images were trimmed to the area common to all exposures of that pointing. The seeing was variable when these data were recorded. It was therefore decided not to construct a mosaic of the entire imaged region, 
but to consider the individual pointings seperately for the photometric analysis. Examples of the final images are shown in Figures 2 and 3, which show the final $K^{\prime}$ images of Pointing 4 and Control Field 2, respectively. The image quality of the Maffei 1 and control field data are mediocre by Mauna Kea standards, and stars in the final images have FWHM between 0.9 and 1.1 arcsec.

\section{RESULTS}

\subsection{Photometric Measurements}

Unresolved stars in Maffei 1 form a nonuniform background that introduces uncertainties in the photometric measurements, especially those of objects within roughly 30 arcseconds of the galactic nucleus. This background galaxy light was removed by applying a $10 \times 10 \operatorname{arcsec}^{2}$ median boxcar filter to the processed images to suppress point sources; the result was subtracted from the unsmoothed data. The brightnesses of individual stars were then measured from the background-subtracted data with the point spread function (PSF) fitting routine ALLSTAR (Stetson \& Harris 1988), using co-ordinates, preliminary stellar brightnesses, and PSFs obtained from routines in the DAOPHOT (Stetson 1987) package.

The photometric calibration was defined using standard stars from Hawarden et al. (2001), which were observed throughout the three night observing run. The standard deviation in the photometric zeropoints obtained from standards stars that were observed during the first night is $\pm 0.1 \mathrm{mag}$ in each bandpass, suggesting that thin cirrus may have been present on that night, which is when data for Pointings 1 and 2 were obtained. For comparison, the standard deviation of the standard star measurements on the second and third nights are \pm 0.06 magnitudes, indicating better photometric stability than during the first night.

The brightnesses of sources in the overlapping portions of Pointings 1 and 2 were compared to check if clouds were present when Maffei 1 was observed during the first night, and the mean differences between the brightness measurements of sources with $J, H$, and $K$ between 16 and 17 are shown in Table 4. The uncertainties in this table are the standard

errors in the mean. Also listed are the mean differences between the brightnesses for sources in the overlapping regions of Pointings 3 and 4, which were observed on the second night. The entries in this table indicate that the pointing-to-pointing photometric stability on each night was within a few hundredths of a magnitude; hence, if thin cirrus was present, then it was uniformly distributed and stable while the observations were recorded. Nevertheless, a comparison between the brightnesses of sources in the overlapping portions of Pointings 
1 and 3 and Pointings 2 and 4 indicates that there is a slight offset between the nightly photometric calibrations. This was greatest in $H$ with $\Delta H=-0.122 \pm 0.012$, while for $J$ and $K$ the differences were $-0.076 \pm 0.012$ and $-0.049 \pm 0.017$. To adjust for this, the brightnesses of sources in Pointings 1 and 2 were shifted by the mean differences to agree with the calibration of Pointings 3 and 4.

Artificial star experiments were run to estimate sample completeness. The artificial stars were assigned colors appropriate for a 'typical' M31 globular cluster, as discussed in $\S 3.4$, after applying an extinction correction $\mathrm{A}_{V}=4.7(\S 1)$. Following the procedures to identify and characterize individual cluster candidates that are discussed in $\S 3$, an artificial star was considered to be recovered only if it was detected in all three bandpasses.

The elevated background levels near the center of Maffei 1 cause incompleteness to become significant at a brighter magnitude than is the case at larger galactocentric distances. To account for the spatially nonuniform completeness function, each pointing was divided into a $3 \times 3$ grid, and the incompleteness in each cell was computed separately. The completeness map for stars with $K$ between 17.75 and 18.25 in Pointing 4 is shown in the upper panel of Figure 4. The completeness fraction, $C$, is lowest in Cell 1, which contains the center of Maffei 1, while $C$ is more or less uniform over the remainder of the field.

The behaviour of $C$ with $K$-band magnitude in three cells that cut diagnonally across Pointing 4 is shown in the lower panel of Figure 4 , and it is evident that $C$ plummets outside of Cell 1 when $K>18$. Therefore, $K=18$ is adopted as the faint limit when searching for globular clusters around Maffei 1. The completeness functions of Pointings 2 and 3 show similar behaviour, while incompleteness sets in at brighter magnitudes in Pointing 1 (§3.2).

\subsection{Near-infrared CMDs of the Central Region of Maffei 1}

The $(K, H-K)$ and $(K, J-K)$ CMDs of the Maffei 1 and control fields are compared in Figures 5 and 6 . The CMDs of the galaxy and control fields are very similar, indicating that the majority of objects near the center of Maffei 1 with $K<18$ are foreground stars. This foreshadows one of the main results of this paper, which is that globular clusters near the center of Maffei 1 make only a minor contribution to the overall source counts in this part of the sky. The $H-K$ and $J-K$ colors of the foreground star sequence also show good field-to-field stability, indicating that large reddening differences on arcminute to

degree scales are abscent. It thus appears that the dense dust clouds found in the vicinity 
of Maffei 1 by Buta \& McCall (1999; 2003) do not affect greatly the near-infrared colors of objects with $K<18$.

Why do the near-infrared colors of objects with $K<18$ show no evidence of the patchy dust absorption that is seen towards Maffei 1? To be sure, the uniform colors are partly due to the decreased sensitivity to extinction in the near-infrared. However, a more important factor is that the majority of sources with $K<18$ along this sight line are probably in front of much of the obscuring material. Buta \& McCall $(1999,2003)$ discuss the origin of the dust that is projected against the inner regions of Maffei 1. Buta \& McCall (1999) point out that many elliptical galaxies have obscuring dust lanes, and so it would not be surprising if this material were ultimately found to belong to Maffei 1. However, Buta \& McCall (2003) argue that the dust is likely in the foreground disk. If this is the case then most of the dust will probably be in the Perseus Arm of the Milky-Way, through which Maffei 1 is viewed. Many of the open clusters within a few degrees of Maffei 1 tend to have distances, based on the entries in the catalogue of Dias et al. (2002), between 2 and $4 \mathrm{kpc}$, and this is adopted in the following discussion as the distance to the Perseus Arm.

Based on the stellar content of the Solar Neighborhood, it can be anticipated that most of the stars along the line of sight will be M dwarfs, and stars of this spectral type that have $K<18$ will be located on the near side of the Perseus Arm or in the interarm region i.e. in front of the region expected to contain the bulk of the dust that is obscuring Maffei 1. For example, an $\mathrm{M} 0 \mathrm{~V}$ star $\left(\mathrm{M}_{K}=+5.2\right.$; Cox 2000$)$ with $K=17$ will be at a distance of $2.3 \mathrm{kpc}$ if there is no extinction, and will be even closer if some extinction is present. M dwarfs with later spectral types that are observed at the same $K$ magnitude will have even smaller distances, as they are intrinsically fainter; for example, an M5 V dwarf $\left(\mathrm{M}_{K}=6.1\right)$ with $K=17$ will be at a distance of at most $1.5 \mathrm{kpc}$. Thus, the majority of stars with $K<18$ along this sight line will likely not be obscured by material in the Perseus Arm. This helps to explain the uniformity in the colors of the stellar sequences, as the localised areas of high extinction along this line of sight are more distant than the majority of stars.

The CMDs of Pointing 1 are peculiar, in that the foreground star sequence truncates near $K=17.5$. The agreement with the photometric measurements in the overlapping portions of Pointing 2 and 3, discussed in $\S 3.1$, indicates that the elevated faint limit in Pointing 1 is not due to clouds. While an extremely thick foreground dust cloud could cause a truncation in the CMD of Pointing 1, most of the dust clouds in the vicinity of Maffei 1 are located in the northern part of the galaxy (Buta \& McCall 1999, 2003, but see also Ford \& Jenner 1971, who trace a dust lane with a width of $\sim 10 \operatorname{arcsec}$ through part of Pointing 1).

The elevated faint limit in Pointing 1 is due to a higher than normal detector noise 
level, which was confirmed by measuring the standard deviation of the counts in a portion of the processed Pointing $1 \mathrm{~K}$-band image that is devoid of objects. The problem appears to be restricted to the Pointing 1 data, and the artificial star experiments confirm that the completeness fraction of the Pointing 1 data are markedly lower when $K>17$ than in the other pointings. While the Pointing 1 data will not be completely discarded, they are of limited use for the globular cluster search, and so will not be considered when computing the specific cluster frequency in $\S 4$.

\subsection{The Identification of a Centrally Concentrated Population of Globular Clusters}

We use the $K$ LFs of the various fields to investigate the field-to-field uniformity of the number counts and conduct an initial reconnaisance for a population of globular clusters, which will show up as an excess population of objects with respect to the source counts in the control fields. The LFs of the various fields, corrected for incompleteness, are compared in Figure 7. The number of objects in the two control fields, which are separated by a degree on the sky, are remarkably similar, indicating that field-to-field variations in the density of foreground stars are abscent at infrared wavelengths when $K<18$. Therefore, the source counts in these fields can be averaged to reduce noise. This uniformity is also seen in the number counts of the Maffei 1 data that, with the exception of Pointing 1 , are remarkably similar over the entire range of brightnesses.

An excess population of objects is present near $K=18$ in Pointings 3 and 4, although the significance of this excess is modest. The statistical significance of the excess population can be enhanced by examining regions that are close to the center of Maffei 1, which is where the density of clusters might be expected to be highest. We further refine the search by restricting the investigation to the magnitude range expected for globular clusters in Maffei 1. Barmby et al. (2001) find that the GCLF of M31 peaks near $M_{K}=-10$, and the LF shown in their Figure 2 indicates that over $90 \%$ of the clusters occur within \pm 2 magnitudes of the peak brightness. If the GCLF of Maffei 1 is like that in M31 then the peak should occur near $K=18.6$ with the adopted distance and reddening, and the vast majority of clusters should have $K>16.6$. Incompleteness is significant in our data when $K>18$ (§3.1), and so the CFHTIR data do not sample the peak of the GCLF, unless the adopted distance modulus is greatly in error.

While it is desireable to extend the search for clusters into the innermost regions of Maffei 1, where the contrast with respect to the foreground stars might be highest, the bright unresolved body of Maffei 1 greatly elevates the noise level near the galaxy center, 
and this consideration sets the innermost radius for our cluster search. A visual inspection of the images indicate that when $r<20$ arcsec the data are affected by noise flucuations, and so the inner radius for the cluster search was fixed at 20 arcsec. The artificial star experiments indicate that the brightness of sources with $K=18$ are not affected by noise when $r>20$ arcsec.

The number of objects with $K$ between 16.6 and 18.0 were counted in four radial intervals centered on the galaxy nucleus: 20 - 30 arcsec, $30-60$ arcsec, $60-90$ arcsec, and $90-120$ arcsec. The raw counts were corrected for incompleteness using the results from the artificial star experiments. The total number of objects in each radial interval in Pointings 2, 3, and 4 are shown in the second column of Table 5. The number of sources expected in each radial interval based on the control field data is shown in the third column of Table 5; the uncertainties in these entries are small because they are based on counts over the total area covered by both control fields. The fourth column of Table 5 shows the difference between the second and third columns.

The entries in the last column of Table 5 indicate that there is an excess number of objects with respect to what is seen in the control field when $r<90$ arcsec. The statistical significance of the excess population in the $30-60$ and $60-90$ arcsec intervals is modest, and this is due to the narrow radial binning interval. The statistical significance can be increased by considering wider radial intervals. For example, between 30 and 90 arcsec the number of excess objects is $17 \pm 9$, while between 20 and $90 \operatorname{arcsec}$ this number is $31 \pm 11$. We identify this excess population as globular clusters in Maffei 1 ; in $\S 4$ these numbers are used to compute $\mathrm{S}_{N}$ for Maffei 1.

\subsection{The $(J-H, H-K)$ TCD and the Near-Infrared Colors of Globular Clusters in Maffei 1}

Foreground stars complicate efforts to identify individual clusters in Maffei 1. Nevertheless, the contrast between the cluster and foreground star populations is sufficient to allow limited conclusions to be drawn regarding the near-infrared colors of clusters in Maffei 1. Such an investigation is aided by the high extinction towards Maffei 1, which facilitates efforts to distinguish between foreground stars and globular clusters on the TCD. In particular, extragalactic sources will be the most heavily reddened objects along the line of sight, while foreground stars will be subject to a range of reddenings; as a result, the heavily reddened Maffei 1 clusters may have observed colors that are redder than those of most stars. Another factor that contributes to efforts to detect Maffei 1 clusters is that the intrinsic near-infrared SEDs of globular clusters differ from those of foreground disk stars, 
the vast majority of which are on the main sequence. $\mathrm{K}$ and $\mathrm{M}$ main sequence stars in the solar neighborhood have unreddened colors $J-H<0.67$, while the $\mathrm{K}$ and $\mathrm{M}$ giants that dominate the near-infrared SEDs of globular clusters can have unreddened $J-H$ colors approaching 1.0 (Bessell \& Brett 1988).

The $(J-H, H-K)$ TCD is a convenient means of examining the broad-band near-infrared SEDs of Maffei 1 globular clusters. The left hand panel of Figure 8 shows the $(J-H, H-K)$ TCD of objects with $K$ between 16.6 and 18.0 in Pointings 2, 3, and 4 that are located between 20 and 90 arcsec from the center of Maffei 1. The composite TCD of the two control fields is shown in the middle panel; the number of objects in the middle panel greatly exceeds that in the left hand panel because the control fields sample a much larger area on the sky than the modest radial interval considered in Maffei 1. Also shown in Figure 8 is the reddening vector and the sequence defined by solar neighborhood $\mathrm{K}$ and M main sequence stars from Bessell \& Brett (1988). The majority of objects in the control fields have $J-H<0.9$, which is what would be expected for $\mathrm{K}$ and $\mathrm{M}$ main sequence stars with $\mathrm{A}_{V} \sim 2$ magnitudes.

The TCDs in Figure 8 have been divided into $0.2 \times 0.2$ magnitude cells to facilitate the statistical analysis of the distribution of sources. The difference between the number of objects in each cell in the Maffei 1 TCD and the number in the corresponding cell in the control field TCD, with the latter scaled to the area searched for clusters in Maffei 1 , is shown in the right hand panel of Figure 8. The number of objects in the Maffei 1 field exceeds that in the control field in all but one of the eight cells, and in three cells the difference is significant at roughly the $2-\sigma$ level or higher.

The cells where there are significant excess populations, which are likely due to globular clusters in Maffei 1, span the full range of colors examined, with $J-H$ between 0.5 and 1.3, and $H-K$ between 0.2 and 0.6. Adopting $\mathrm{A}_{V}=4.7$, the color excesses computed from the Rieke \& Lebofsky (1985) reddening curve are $E(H-K)=0.30$ and $E(J-H)=0.50$. Thus, significant excess populations occur in cells with $(J-H)_{0}$ between 0.0 and 0.8 and $(H-K)_{0}$ between -0.1 and 0.3 . For comparison, data from Barmby et al. (2000) and Barmby, Huchra, \& Brodie (2001) indicate that the majority of globular clusters in M31 have $(J-H)_{0}=0.6 \pm 0.2$ and $(H-K)_{0}=0.1 \pm 0.1$. The globular clusters in Maffei 1 thus cover a range of intrinsic colors that is at least as large as that seen among M31 clusters. The clusters in the cell in the lower left hand corner of the TCD have infrared colors that are bluer than those of M31 clusters, and we note that 2 of the 20 cluster candidates identified by Buta \& McCall (2003) in Maffei 1 have $(R-I)_{0} \sim 0$. While the nature of these objects is a matter of speculation, one possible interpretation is that they might be young clusters. 
The five objects in the cell with $J-H>1.1$ and $H-K>0.4$ are of particular interest, as no objects with this color are seen in the control fields. After correcting for extinction, the objects in this cell have intrinsic colors that fall within the range seen among members of the M31 cluster system, and so they do not have peculiar near-infrared SEDs. Given the absence of objects with similar colors in the control field, we expanded the search for objects in this cell to cover all of Pointings 2, 3, and 4, and an additional 8 objects were found.

The spatial distribution of these red objects provides a means of checking if they are truely clusters, as the density of clusters is expected to rise towards smaller galactocentric radii. The artificial star experiments indicate that the CFHTIR data are most sensitive to sources more than $\sim 30$ arcsec from the center of Maffei 1 . Nevertheless, 5 of the 13 cluster candidates identified throughout the area imaged with CFHTIR are located within 30 arcsec of the nucleus, suggesting that the cluster candidates are concentrated near the nucleus of Maffei 1. The spatial distribution of the red objects is examined in Figure 9, where the differential and integrated counts of these objects in 4 radial intervals are compared with the predictions for a uniformly distributed population; the number counts have been corrected for incompleteness. It is apparent that the majority of sources found on the TCD are concentrated towards the nucleus, with a statistical significance that is slightly in excess of the $2-\sigma$ level in the innermost bin. Therefore, we conclude that the spatial distribution of the very red objects on the TCD is consistent with them being globular clusters.

The main result of this section is that the clusters around Maffei 1 span a large range of near-infrared colors, and this can be checked by investigating the colors of globular clusters identified using independent criteria. Buta \& McCall (2003) used the non-stellar appearance of objects in WFPC2 images to identify clusters near the center of Maffei 1. The extended nature of an object is a criterion that is systematically different from that employed here, and should be unbiased in terms of color. Five of the Buta \& McCall (2003) candidate clusters were detected in all three filters in the CFHTIR data, and the near-infrared brightnesses and colors of these objects are listed in Table 6 . The $K$ magnitudes of these clusters place them at the bright end of the GCLF. One of these objects falls outside of the region investigated in Figure 8, while 3 of the remaining 4 clusters fall in regions of the near-infrared TCD where a statistically significant excess with respect to the control field counts were found. The clusters identified by Buta \& McCall (2003) thus span a wide range of near-infrared colors, in agreement with the analysis of Figure 8. 


\subsection{Very Red Objects}

There are a modest number of objects in the Maffei 1 and control fields with $J-H$ and $H-K$ colors that are much redder than the box limits marked in Figure 8 . There are 7 such objects in Pointings 1 - 4, and 3 such objects in the two control fields. The number density of these objects is therefore the same in the control and Maffei 1 fields, indicating that they are not associated with Maffei 1 . These objects tend to have only a modest object-to-object dispersion in $H-K$ and $J-H$ colors, with mean values $\overline{H-K}=0.94 \pm 0.13$ and $\overline{J-H}=1.30 \pm 0.15$; the quoted uncertainties are the standard deviations about the mean.

The projected density of the very red objects, which have $K$ between 16.8 and 17.8 with a mean near $K=17.2$, is $\sim 500$ degree $^{-2}$. For comparison, the density of galaxies with $K_{0}=16.7$ is $\sim 800$ degree $^{-2}(0.5 \text { magnitude })^{-1}$ (e.g. McCracken et al. 2000), and so the majority of very red objects in our data are likely galaxies. The near-infrared colors of the very red objects are consistent with this claim. Fioc \& Rocca-Volmerange (1999) investigated the integrated near-infrared colors of nearby galaxies, and found that elliptical and spiral galaxies typically have $J-H \sim 0.75$ and $H-K \sim 0.25$. Now, if the very red objects are galaxies at intermediate redshift then k-corrections will be significant. In fact, Cowie et al. (1996) tabulated redshifts for galaxies in two fields, and the entries in their

Table 1 indicate that galaxies with $K_{0}$ between 16.3 and 17.3 have a tight range of redshifts, with $\langle z\rangle=0.33$ and a standard deviation \pm 0.14 . Mannucci et al. (2001) computed $\mathrm{k}$-corrections in the near-infrared, and the data in their Figure 7 indicate that for $\mathrm{z}=0.3$ the k-corrections in $J, H$, and $K$ are $\mathrm{k}_{\text {Corr }}^{J}=0.1, \mathrm{k}_{\text {Corr }}^{H}=0.0$, and $\mathrm{k}_{\text {Corr }}^{K}=-0.6$ independent of morphology. Thus, a 'typical' $\mathrm{z}=0.3$ galaxy when viewed through $\mathrm{A}_{V}=4.7$ magnitudes of extinction would have $J-H \sim 1.35$ and $H-K \sim 1.15$; these are in good agreement with the colors of the very red objects in the Maffei 1 and control fields.

A modest number of the very red objects may also be L dwarfs. Kirkpatrick et al. (1999; 2000) find $62 \mathrm{~L}$ dwarfs to the 2MASS completeness limit of $K \sim 14.3$ in an area of 371 degree $^{2}$, and so the projected density on the sky is 0.2 degree $^{-2}$. Adjusting for the greater depth of our observations and assuming a uniform spatial distribution for L dwarfs in the solar neighborhood, the expected L dwarf density based on the Kirkpatrick et al. (2000) survey is 50 degree $^{-2}$ to our faint limit of $K=18$, and so $\sim 1$ of the very red objects may be an L dwarf. In fact, the near-infrared SEDs of the very red objects are consistent with some fraction being $\mathrm{L}$ dwarfs. This is demonstrated in Figure 10, which shows the TCD of the L dwarfs discovered by Kirkpatrick et al. (1999, 2000) and the very red objects found here. If the objects in our data are L dwarfs then they would be intrinsically faint, and hence close to the Sun. Consequently, they will not be viewed through the 4.7 
magnitudes of absorption in $V$ that affects Maffei 1, and the reddening correction will be smaller than that indicated by the reddening vector in Figure 10.

The K-band spectra of L dwarfs show deep absorption bands due to $\mathrm{H}_{2} \mathrm{O}$ and $\mathrm{CO}$. These features are very wide, spanning wavelength intervals in excess of $0.1 \mu \mathrm{m}$ (e.g. Figure 5 of Kirkpatrick et al. 1999). There are narrow-band filters that measure the strengths of both of these molecules in the $K$-band, and a narrow-band filter survey would provide a means of discriminating between L dwarfs and distant galaxies in the vicinity of Maffei 1 .

\section{THE SPECIFIC CLUSTER FREQUENCY NEAR THE CENTER OF MAFFEI 1}

The data are limited to $K=18$, and so any clusters that are fainter than this are missed. The number of missing clusters can be estimated by assuming a shape for the GCLF. We adopt the M31 $K$-band GCLF of Barmby et al. (2001). However, their LF is affected by incompleteness at the faint end. To correct for this, the LF was assumed to be symmetric about the GCLF peak, and the entries at the bright end of the LF were reflected about the peak to fill in the faint end. Integrating the result indicates that a search for globular clusters in Maffei 1 that is limited to $K$ between 16.6 and $18.0\left(\mathrm{M}_{K}\right.$ between -12.0 and -10.6 ) would detect only the brightest $27 \%$ of the total cluster population.

Given that the magnitude range considered in $\S 3.3$ includes $27 \%$ of the cluster population, then the total number of clusters in Pointings 2, 3, and 4 located between 20 and 90 arcsec of the center is $(31 \pm 11) / 0.27=115 \pm 41$. Using the $V$-band light profile for Maffei 1 shown in Figure 24 of Buta \& McCall (1999), and not counting the area sampled with Pointing 1, we find that $\mathrm{M}_{V}=-19.9$ between 20 and 90 arcsec of the nucleus if the distance modulus is $28.1 \pm 0.2$ and $\mathrm{A}_{V}=4.7 \pm 0.2$. Consequently, the specific cluster frequency near the center of Maffei 1, including the uncertainties in the distance modulus and foreground absorption, is $\mathrm{S}_{N}=1.3 \pm 0.6$.

\section{DISCUSSION \& SUMMARY}

Near-infrared images recorded with the CFHTIR imager have been used to search for globular clusters in the central $6 \times 6 \operatorname{arcmin}^{2}$ of Maffei 1 , which is one of the nearest large elliptical galaxies. The area surveyed includes more than half of the integrated light from the galaxy. The cluster content is investigated in a statistical manner by searching for an excess population of sources with respect to foreground disk stars. This is done near the 
center of the galaxy, where the projected concentration of clusters is greatest. The specific cluster frequency, $\mathrm{S}_{N}$, is $1.3 \pm 0.6$ between 20 and 90 arcsec of the galaxy center.

\subsection{Uncertainties in the Specific Cluster Frequency}

The $\mathrm{S}_{N}$ computed here holds for the inner regions of the galaxy, and is not a global value. In fact, $\mathrm{S}_{N}$ changes with radius in elliptical galaxies, in the sense of becoming larger with increasing radius (e.g. Blakeslee \& Tonry 1995; Harris 1991). Because of such a possible gradient in $\mathrm{S}_{N}$, our local $\mathrm{S}_{N}$ for Maffei 1 should be regarded as a lower limit to the global value, at least to the extent that the spatial distribution of clusters in Maffei 1 may be similar to that in the Coma and Virgo elliptical galaxies considered by Blakeslee \& Tonry (1995) and Harris (1991).

The current observations sample only the bright end of the cluster population in Maffei 1, and to compute the total number of clusters the GCLF of M31 was adopted. Dynamical processes, which are expected to be most effective in the dense central regions of galaxies, can cause globular clusters to be disrupted over time. These processes will preferentially destroy low mass, extended clusters. The shape of the GCLF near the galaxy center may thus change over time (e.g. Vesperini 2000; 2001), and may vary from galaxy-to-galaxy depending on stellar density and the dynamical state of the cluster system. While we have no means of monitoring the extent of this evolution among clusters in Maffei 1 , we note that the innermost regions of the galaxy were avoided when measuring $S_{N}$.

There are also practical problems inherent to studies of the central regions of galaxies. Spurious noise sources may masquerade as clusters near the nucleus of Maffei 1, and the artificial star experiments indicate that these flucuations affect the brightnesses of real objects in this region. In an effort to avoid these problems, we did not search for clusters in the central 20 arcsec of Maffei 1, where noise flucuations are clearly present (e.g. Figure 2).

Ideally, the impact of these effects could be monitored directly by investigating how $\mathrm{S}_{N}$ changes with radius in Maffei 1. However, foreground star contamination limits our ability to do this. In an effort to assess radial effects, $\mathrm{S}_{N}$ was computed using only the candidate clusters detected between 30 and 90 arcsec of the galaxy center. We find that $\mathrm{S}_{N}=0.8 \pm 0.5$, which is not statistically different from $\mathrm{S}_{N}$ computed for the region between 20 and 90 arcsec from the nucleus. 


\subsection{The Colors of Clusters in Maffei 1}

Buta \& McCall (2003) found that the candidate clusters in their sample tend to have $R-I$ colors that are redder, and have a larger scatter about the mean $R-I$ value, than expected if the Maffei 1 and Galactic globular cluster systems are similar. While our data confirm that the globular clusters around Maffei 1 span a broad range of near-infrared colors, there is no evidence that they are dominated by very red clusters. Indeed, the five clusters that we have detected from the Buta \& McCall (2003) sample have a broad range of near-infrared colors, with some falling near the blue end of what is seen among M31 clusters, and some falling near the red end. This is consistent with the near-infrared colors of the larger population of sources detected between 20 and 90 arcsec of the center of Maffei 1 (§3.4). We emphasize that the reddening towards Maffei 1 is uncertain (e.g. $\S 1$ ), and that the $R-I$ colors computed by Buta \& McCall (2003) are more prone to uncertainties in the reddening than the near-infrared colors measured here.

\subsection{Comparisons With Other Systems}

How does the $\mathrm{S}_{N}$ of Maffei 1 compare with what is seen other elliptical galaxies? There is a well documented broad spread in $\mathrm{S}_{N}$ values among elliptical galaxies. Harris (1991) lists $\mathrm{S}_{N}$ for 27 elliptical galaxies, and the entries in his Table 1 range from $\mathrm{S}_{N}=0.4 \pm 0.3$ (NGC 3557) to $14 \pm 3$ (NGC 4486). The mean $\mathrm{S}_{N}$ in the Harris (1991) elliptical galaxy sample is 5.1, with a standard deviation of \pm 3.5 . Kundu \& Whitmore (2001) measured $\mathrm{S}_{N}$ in 28 elliptical galaxies, and found that $\mathrm{S}_{N}$ ranged between $0.5 \pm 0.1$ (NGC 3379) to $17.4 \pm 22$ (NGC 5982), with a mean of $2.4 \pm 1.8$. The CFHTIR data indicate that $\mathrm{S}_{N}$ near the center of Maffei 1 falls near the lower end of global $S_{N}$ values for elliptical galaxies. This suggests that Maffei 1 likely experienced a star-forming history that is not atypical for elliptical galaxies, and that the SGMCs needed to form globular clusters evidently occured in comparatively modest numbers during the early history of the galaxy and any progenitor systems.

The broad range in $\mathrm{S}_{N}$ among elliptical galaxies is due in part to environmental effects, in the sense that the elliptical galaxies with the smallest $\mathrm{S}_{N}$ s tend to be those in low density environments (Harris 1991). Cen A (NGC 5128) is a well-studied elliptical galaxy that has a similar environment, distance, and bright red stellar content to Maffei 1 (e.g. Davidge 2002). However, unlike Maffei 1, Cen A contains a prominent dust lane and outer shell structures (e.g. Malin, Quinn, \& Graham 1983) that are signatures of a recent merger. The specific cluster frequency in Cen $\mathrm{A}$ is $\mathrm{S}_{N}=1.4 \pm 0.2$ (Harris, Harris, \& Geisler 2004). While we caution that the cluster sample used by Harris et al. (2004) is dominated by 
objects in the outer regions of the galaxy, as the dust lane that slices through the center of the galaxy makes it difficult to detect objects within a few arcmin of the nucleus, the global $\mathrm{S}_{N}$ in NGC 5128 is in excellent agreement with what we have measured near the center of Maffei 1; the list of similar characteristics of NGC 5128 and Maffei 1 thus may include the specific frequency of globular clusters.

\subsection{Future Work}

Clearly, it is of interest to expand the sample of clusters in Maffei 1 to larger radii, and to go deeper. Because of the high density of foreground stars, it will be helpful to use criteria such as morphology, radial velocity, and spectral characteristics - especially the depths of absorption features that are sensitive to surface gravity - to identify individual clusters out to large galactocentric radii. Work of this nature would most easily be done in the infrared, given the substantial line of site extinction towards Maffei 1. Deeper samples are of particular interest, since these will allow the turn-over in the GCLF, which is a standard candle, to be identified. Indeed, the various distance moduli listed in Table 1 span $0.8 \mathrm{dex}$, and the detection of the turn-over in the GCLF will help resolve any uncertainty in the true distance modulus of Maffei 1. 


\begin{tabular}{lll}
\hline \hline Method & $(m-M)_{0}$ & Reference \\
\hline Surface Brightness Flucuations & $28.1 \pm 0.25$ & Luppino \& Tonry (1993) \\
Brightest AGB Stars & $28.2 \pm 0.3$ & Davidge \& van den Bergh (2001) \\
Fundamental Plane $/ D_{n}-\sigma$ & $27.4 \pm 0.2$ & Fingerhut et al. (2003) \\
\hline
\end{tabular}

Table 1: Distance Modulus Estimates for Maffei 1 


\begin{tabular}{lcl}
\hline \hline Method & $\mathrm{A}_{V}$ & Reference \\
\hline Integrated color & $5.1 \pm 0.2$ & Buta \& McCall (1983) \\
Color of AGB Stars & $4.5 \pm 0.8$ & Davidge (2002) \\
$\mathrm{Mg}_{2}$ - Color relation & $4.67 \pm 0.19$ & Fingerhut et al. (2003) \\
\hline
\end{tabular}

Table 2: Extinction Estimates for Maffei 1 


\begin{tabular}{lll}
\hline \hline $\begin{array}{l}\text { Field } \\
\#\end{array}$ & RA (J2000) & Dec (J2000) \\
\hline 1 & $02: 36: 35.4$ & $+59: 09: 19$ \\
2 & $02: 36: 35.4$ & $+60: 09: 19$ \\
\hline
\end{tabular}

Table 3: Co-ordinates of the Control Fields 


\begin{tabular}{lccc}
\hline \hline $\begin{array}{l}\text { Pointings } \\
\text { Compared }\end{array}$ & $\Delta J$ & $\Delta H$ & $\Delta K$ \\
\hline $1 \& 2$ & $0.017 \pm 0.008$ & $0.040 \pm 0.017$ & $0.012 \pm 0.020$ \\
$3 \& 4$ & $0.007 \pm 0.017$ & $-0.006 \pm 0.012$ & $0.048 \pm 0.014$ \\
\hline
\end{tabular}

Table 4: Comparison of Photometric Measurements 


\begin{tabular}{lccc}
\hline \hline $\begin{array}{l}\text { Radial Interval } \\
(\operatorname{arcsec})\end{array}$ & $\mathrm{N}_{\text {Maffei1 }}$ & $\mathrm{N}_{\text {Control }}$ & $\Delta \mathrm{N}$ \\
\hline $20-30$ & $20.0 \pm 6.0$ & $5.7 \pm 0.3$ & $14 \pm 6$ \\
$30-60$ & $31.0 \pm 5.6$ & $23.0 \pm 1.2$ & $8 \pm 6$ \\
$60-90$ & $47.0 \pm 6.9$ & $38.3 \pm 2.0$ & $9 \pm 7$ \\
$90-120$ & $59.0 \pm 7.7$ & $53.7 \pm 2.9$ & $5 \pm 8$ \\
\hline
\end{tabular}

Table 5: The Excess Number of Objects with $K$ Between 16.6 and 18.0 in Three Radial Intervals 


\begin{tabular}{lccc}
\hline \hline Cluster \# & $K$ & $J-H$ & $H-K$ \\
\hline 1 & 16.467 & 1.167 & 0.824 \\
12 & 16.867 & 0.763 & 0.326 \\
14 & 16.289 & 0.798 & 0.225 \\
17 & 16.715 & 1.132 & 0.543 \\
18 & 17.605 & 0.929 & 0.188 \\
\hline
\end{tabular}

Table 6: Near-Infrared Photometry of Globular Cluster Candidates Identified by Buta \& McCall (2003) 


\section{REFERENCES}

Barmby, P., Huchra, J. P., \& Brodie, J. P. 2001, AJ, 121, 1482

Barmby, P., Huchra, J. P., Brodie, J. P., Forbes, D. A., Schroder, L. L., \& Grillmair, C. J. 2000, AJ, 119, 727

Beasley, M. A., Kawata, D., Pearce, F. R., Forbes, D. A., \& Gibson, B. K. 2003, ApJ, 596, L187

Bekki, K., \& Freeman, K. C. 2003, MNRAS, 346, L11

Bessell, M. S., \& Brett, J. M. 1988, PASP, 100, 1134

Blakeslee, J. P., \& Tonry, J. L. 1995, ApJ, 442, 579

Buta, R., \& McCall, M. L. 1999, ApJS, 124, 33

Buta, R., \& McCall, M. L. 2003, AJ, 125, 1150

Cowie, L. L., Songaila, A., Hu, E. M., \& Cohen, J. G. 1996, AJ, 112, 839

Cox, A. N. 2000, Astrophysical Quantities, Fourth Edition, (Springer-Verlag: New York)

Davidge, T. J. 2002, AJ, 124, 2012

Davidge, T. J., \& van den Bergh, S. 2001, ApJ, 553, L133

Dias, W. S., Alessi, B. S., Moitinho, A., \& Lepine, J. R. D. 2002, A\&A, 389, 871

Fingerhut, R. L., McCall, M. L., De Robertis, M., Kingsburgh, R. L., Komljenovic, M., Lee, H., \& Buta, R. J. 2003, ApJ, 587, 672

Fioc, M., \& Rocca-Volmerange, B. 1999, A\&A, 351, 869

Forbes, D. A., \& Forte, J. C. 2001, MNRAS, 322, 257

Ford, H. C., \& Jenner, D. C. 1971, ApJ, 165, L1

Harris, W. E. 1991, ARAA, 29, 543

Harris, W. E., \& Pudritz, R. E. 1994, ApJ, 429, 177

Harris, W. E., \& van den Bergh, S. 1981, AJ, 86, 1627

Harris, G. L. H., Harris, W. E., \& Geisler, D. 2004, AJ, 128, 723

Hawarden, T. G., Leggett, S. K., Letawsky, M. B., Ballantyne, D. R., \& Casali, M. M. 2001, MNRAS, 325, 563

Kirkpatrick, J. D. et al. 1999, ApJ, 519, 802

Kirkpatrick, J. D. et al. 2000, AJ, 120, 447

Kundu, A., \& Whitmore, B. C. 2001, AJ, 121, 2950 
Luppino, G. A., \& Tonry, J. L. 1993, ApJ, 410, 81

Malin, D. F., Quinn, P. J., \& Graham, J. A. 1983, ApJ, 272, L5

Mannucci, F., Basile, F., Poggianti, B. M., Cimatti, A., Daddi, E., Pozzerri, L., \& Vanzi, L. 2001, MNRAS, 326, 745

McCracken, H. J., Metcalfe, N., Shanks, T., Campos, A., Gardner, J. P., \& Fong, R. 2000, MNRAS, 311, 707

Meylan, G., Sarajedini, A., Jablonka, P., Djorgovski, S. G., Bridges, T., \& Rich, R. M. 2001, AJ, 122, 830

Minniti, D. 1995, AJ, 109, 1663

Mizutani, A., Chiba, M., \& Sakamoto, T. 2003, ApJ, 589, L89

Rieke, G. H., \& Lebofsky, M. J. 1985, ApJ, 288, 618

Solomon, P. M., \& Rivolo, A. R. 1989, ApJ, 339, 919

Stetson, P. B. 1987, PASP, 99, 191

Stetson, P. B., \& Harris, W. E. 1988, AJ, 96, 909

Strader, J., Brodie, J. P., \& Forbes, D. A. 2004, AJ, 127, 3431

van den Bergh, S., \& Mackey, A. D. 2004, MNRAS, 354, 713

Vesperini, E. 2000, MNRAS, 318, 841

Vesperini, E. 2001, MNRAS, 322, 247

Zinn, R. 1985, ApJ, 293, 424 


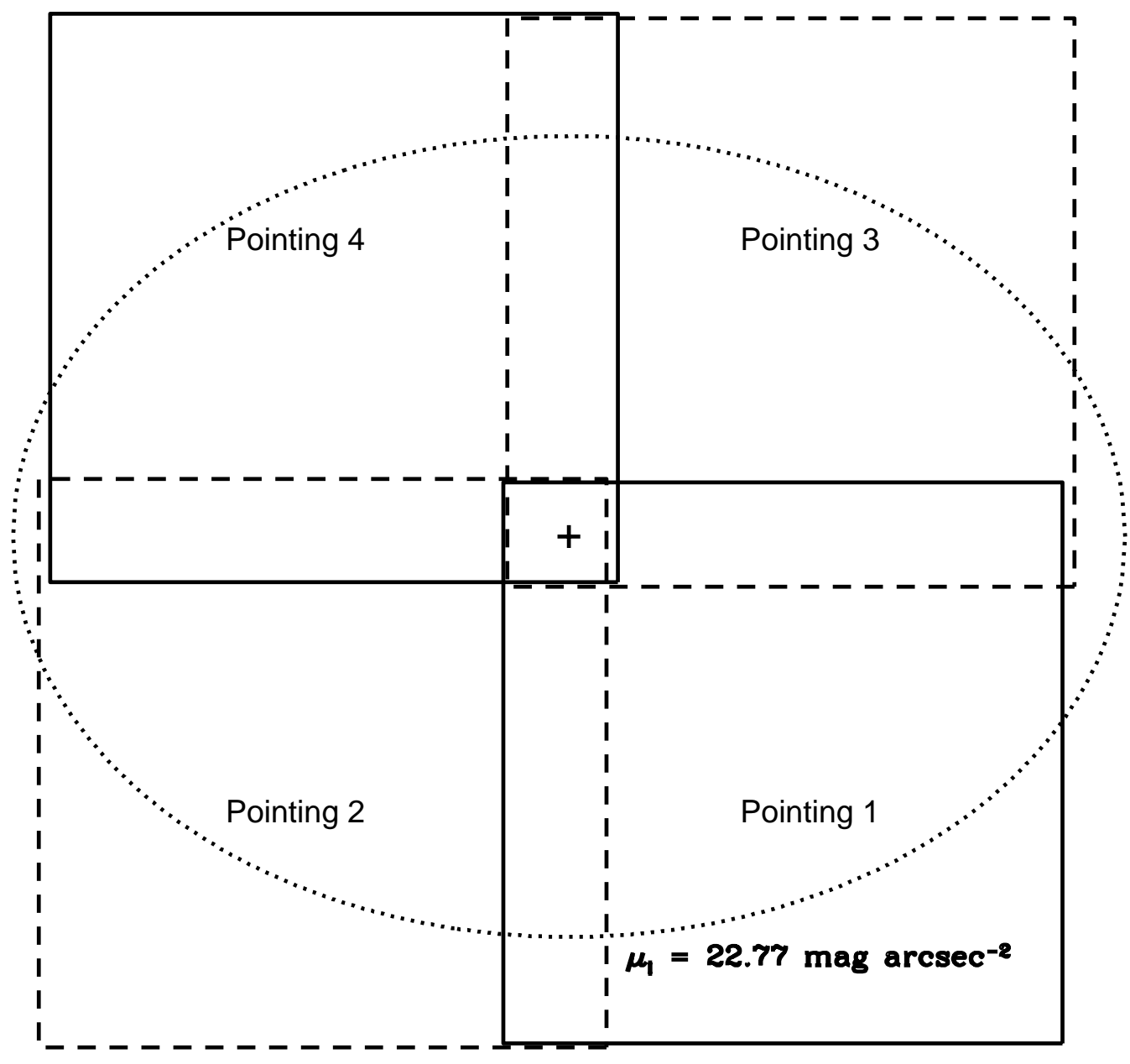

Fig. 1. - The geometry of the pointings used to map the central $6 \times 6 \operatorname{arcmin}^{2}$ of Maffei 1 . North is at the top, and East is to the left. The cross marks the center of Maffei 1, while the dotted line indicates the $\mu_{I}=22.77 \mathrm{mag} \operatorname{arcsec}^{-2}$ isophote, which contains half of the integrated light from Maffei 1 (Buta \& McCall 1999). 


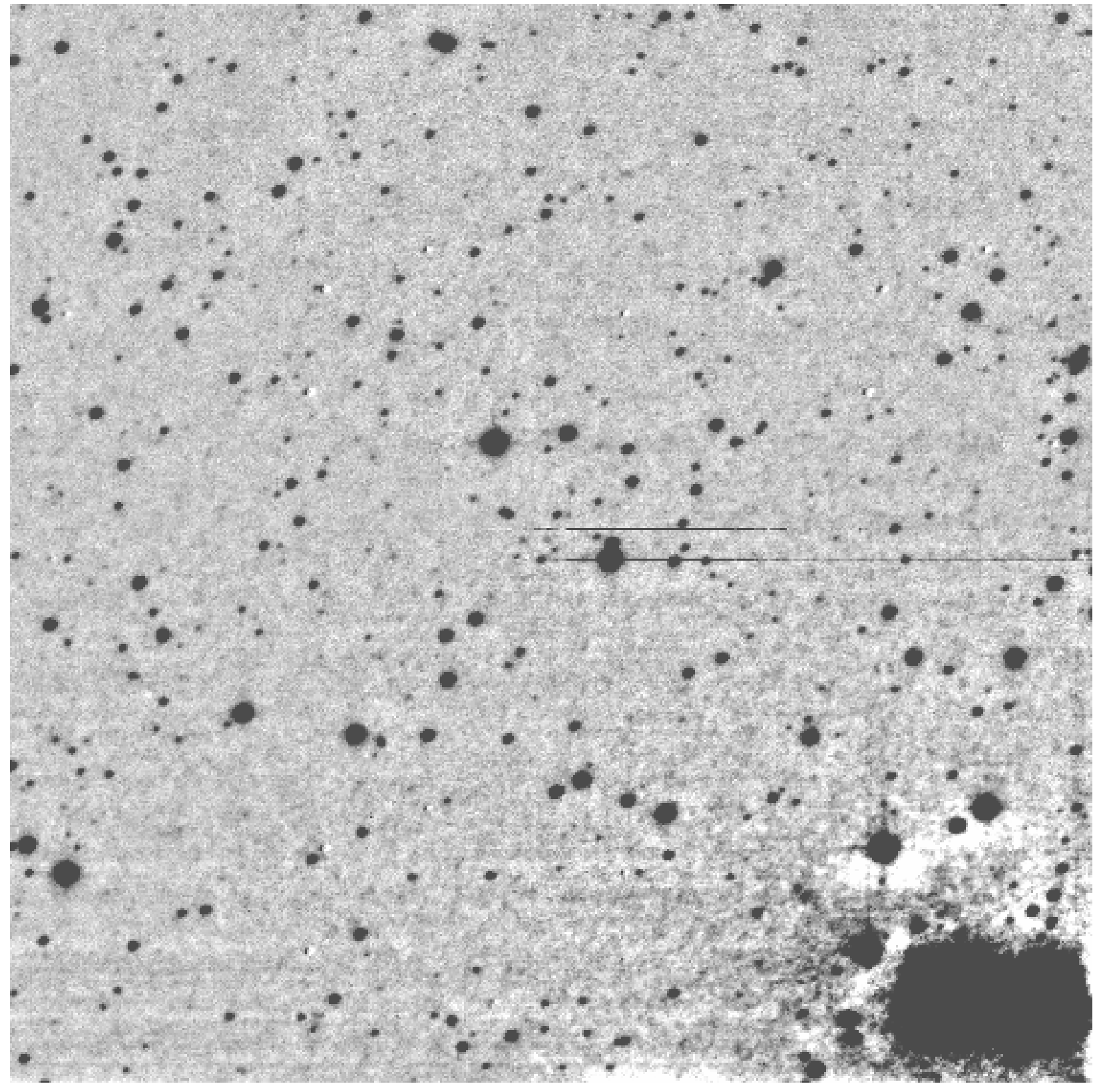

Fig. 2.- The final processed $K^{\prime}$ image of Pointing 4, which covers $3.6 \times 3.6$ arcmin $^{2}$. North is at the top, and East is to the left. The center of the galaxy is in the lower right hand corner. The unresolved body of Maffei 1 has been removed using the procedure discussed in Section 3.1. The majority of objects in this image are foreground stars. 


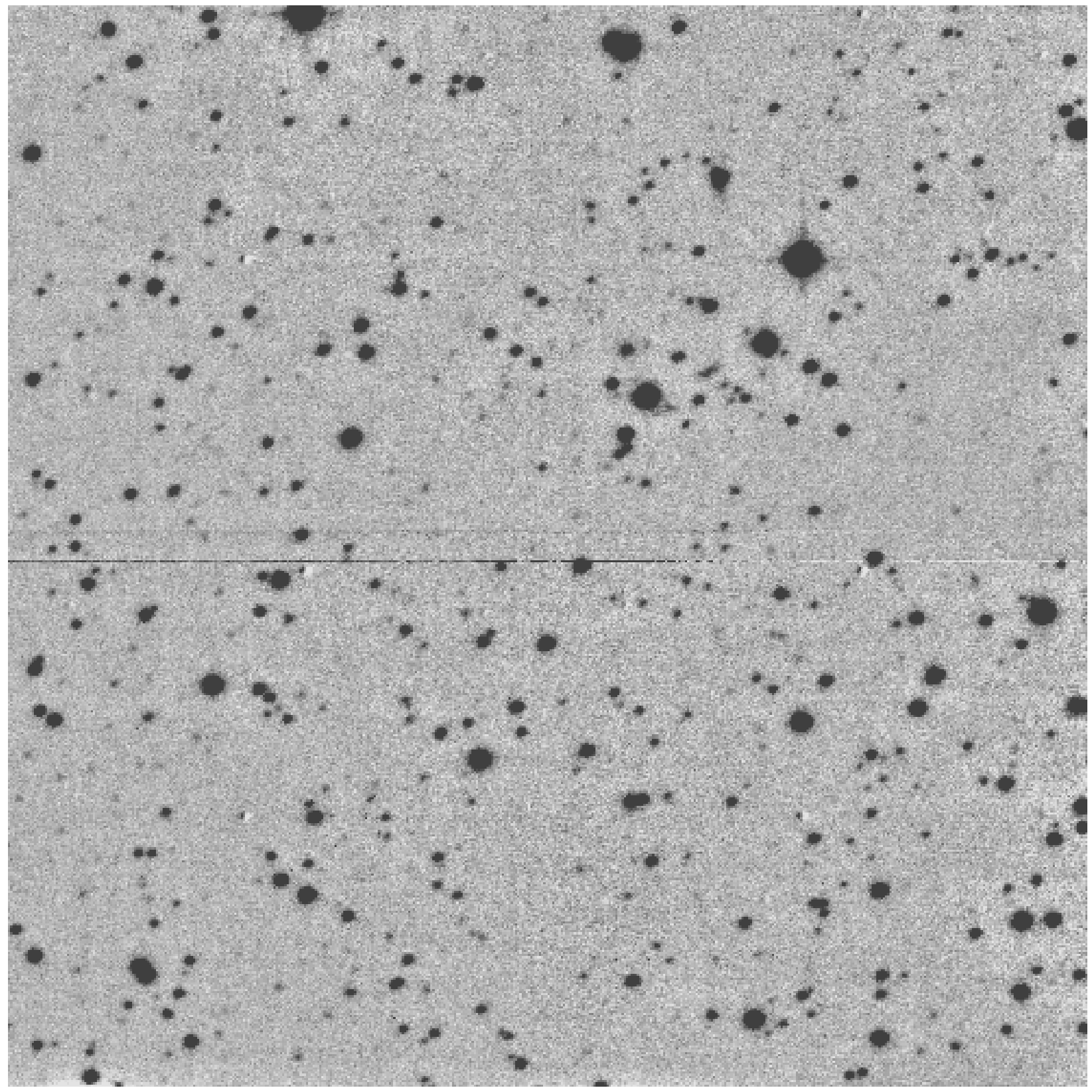

Fig. 3. - The final processed $K^{\prime}$ image of Control Field 2, which covers $3.6 \times 3.6$ arcmin ${ }^{2}$. North is at the top, and East is to the left. Note that the density of objects in this field is roughly comparable to that in Figure 2, indicating that foreground disk stars dominate source counts near Maffei 1. 


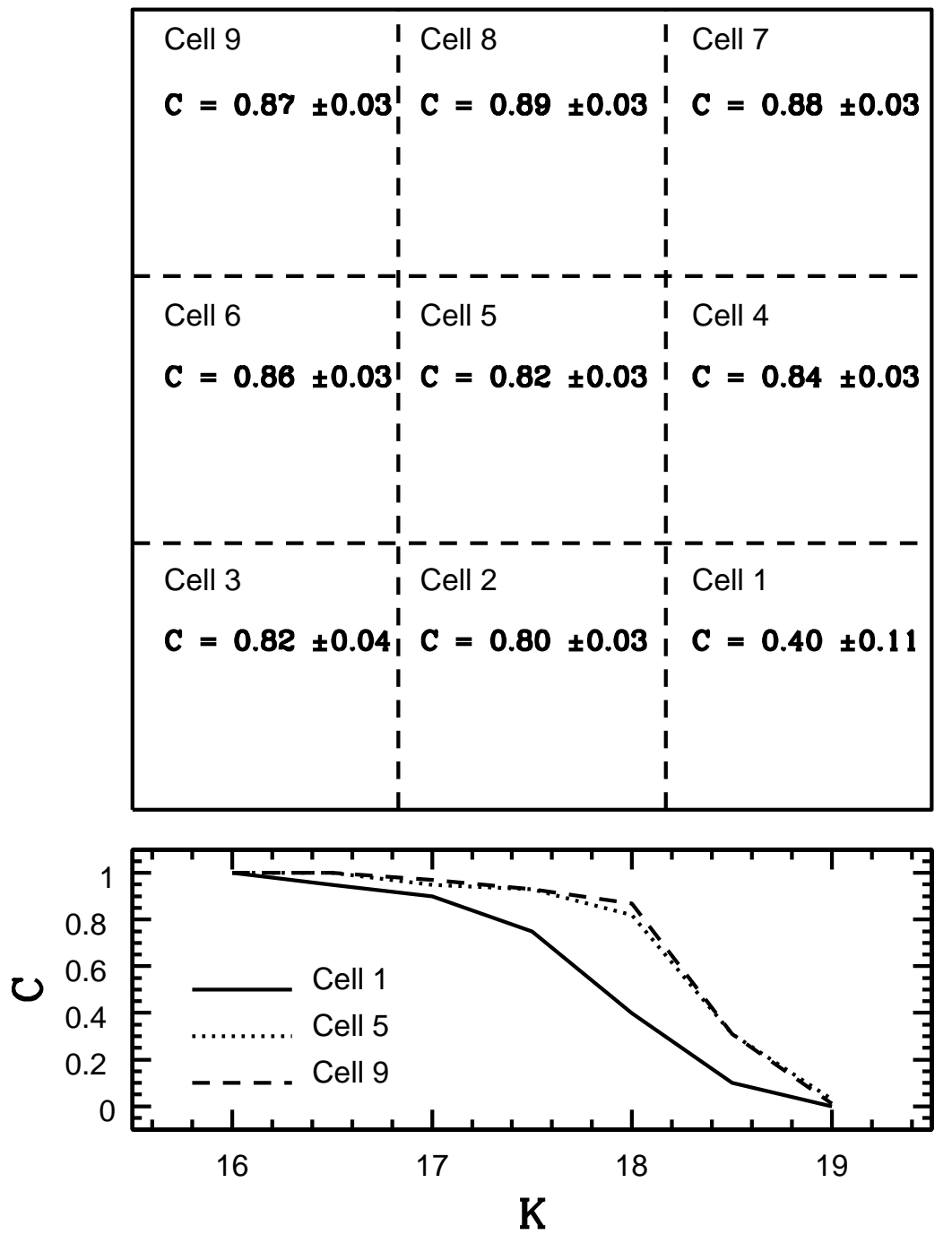

Fig. 4.- The behaviour of the completeness fraction $C$, which is the number of artificial stars recovered in all three filters divided by the total number that were added per magnitude interval, as a function of location and $K$ magnitude in Pointing 4. The top panel shows the spatial distribution of $C$ for sources in Pointing 4 with $K$ between 17.75 and 18.25. The cells have equal area, and are roughly 1.2 arcmin on a side. The center of Maffei 1 is located in Cell 1 , and the elevated noise level resulting from the high surface brightness central regions of Maffei 1 causes $C$ in Cell 1 to be lower than elsewhere in Pointing 4. The lower panel shows $C$ as a function of $K$ in Cells 1, 5 , and 9, which cut diagonally across Pointing 4. Note that the completeness fraction drops rapidly when $K>18$ in Cells 5 and 9. 

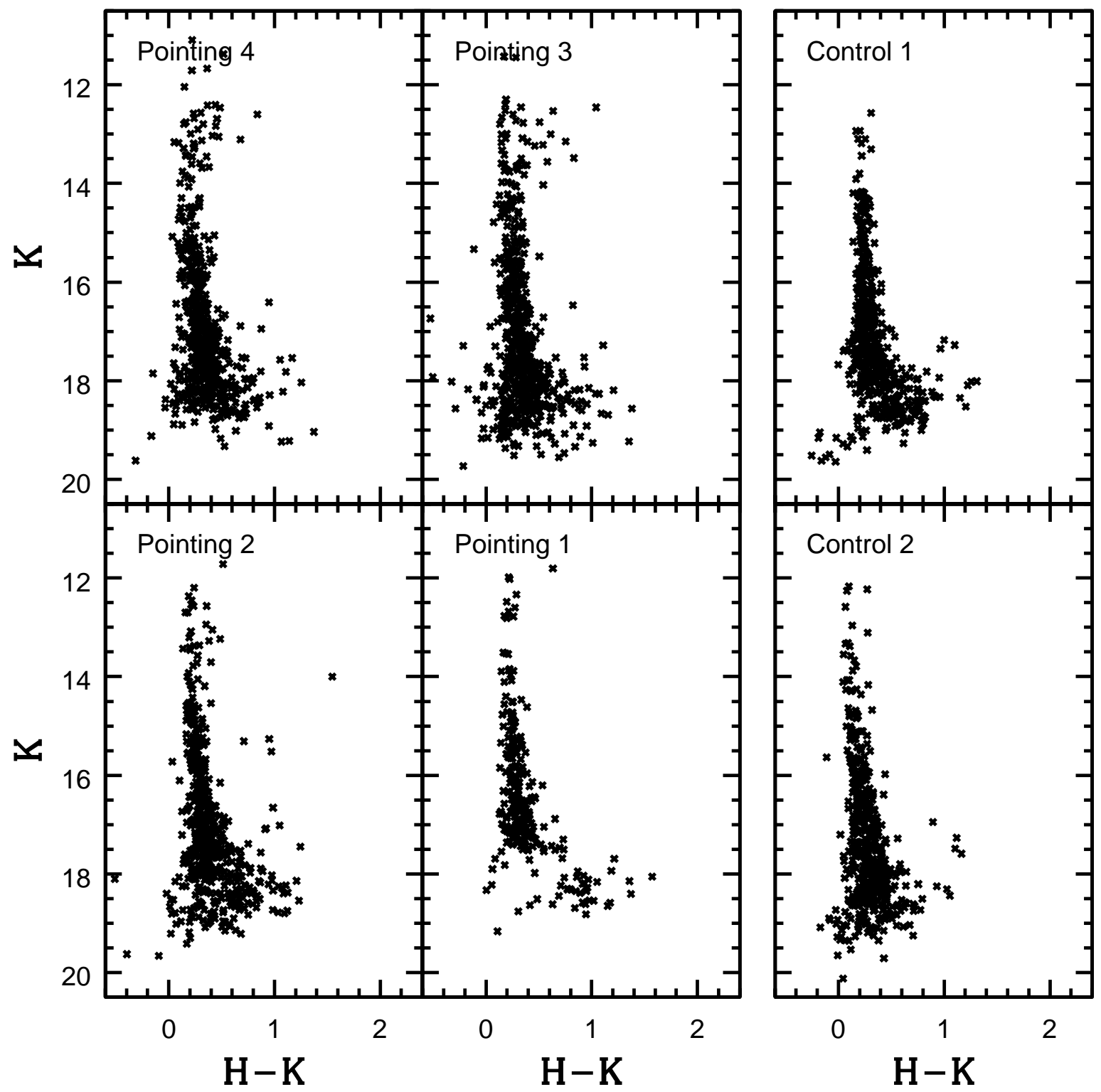

Fig. 5.- The $(K, H-K)$ CMDs of the Maffei 1 and control fields. The Maffei 1 CMDs are positioned according to the geometry indicated in Figure 1. Note the similarities between the Maffei 1 and control field CMDs, indicating that the vast majority of objects with $K<18$ are stars in the foreground disk. 

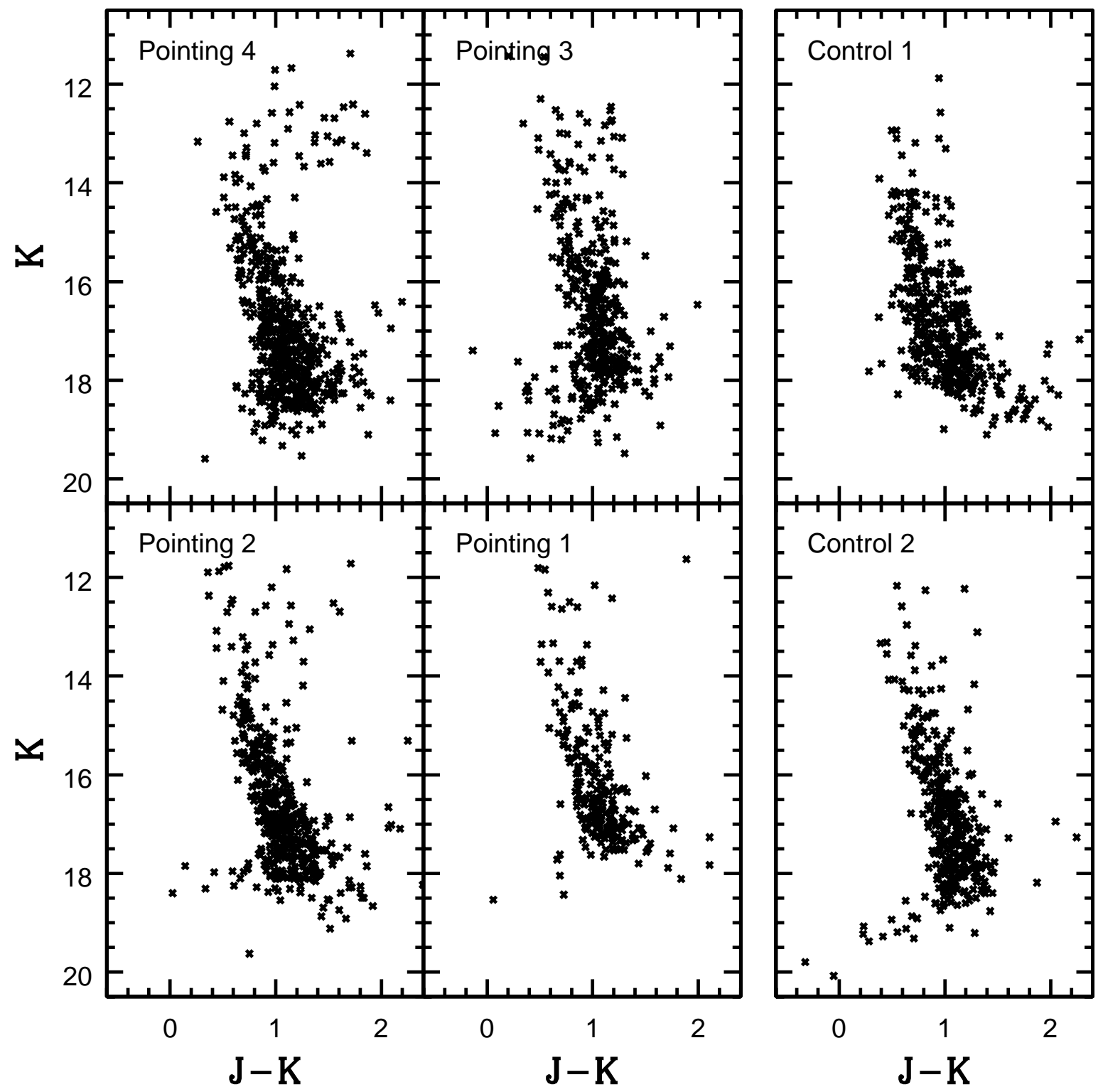

Fig. 6. - The same as Figure 5, but for the $(K, J-K)$ CMDs. 


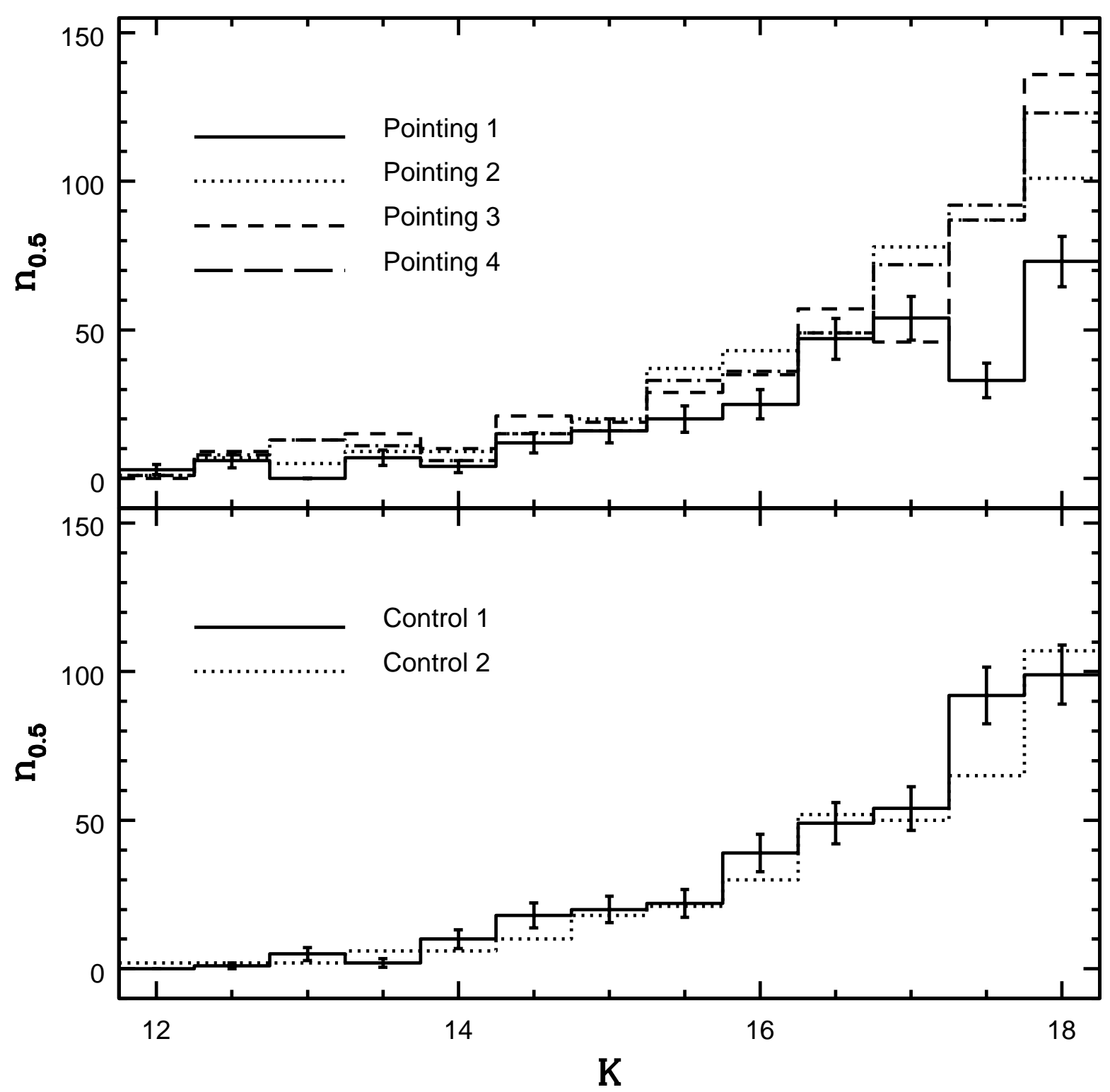

Fig. 7.- The $K$ LFs of the Maffei 1 and control fields. The error bars show the uncertainties in the Pointing 1 (top panel) and Control 1 (lower panel) number counts. $\mathrm{n}_{0.5}$ is the number of objects per 0.5 magnitude interval in $K$, corrected for incompleteness. There is excellent agreement between the number counts in the two control fields, indicating that the foreground stars are uniformly distributed over 1 degree angular scales in this part of the sky. Note that Pointings 3 and 4 appear to have an excess number of sources near $K=18$ when compared with the control fields; these excess sources are globular clusters in Maffei 1. 


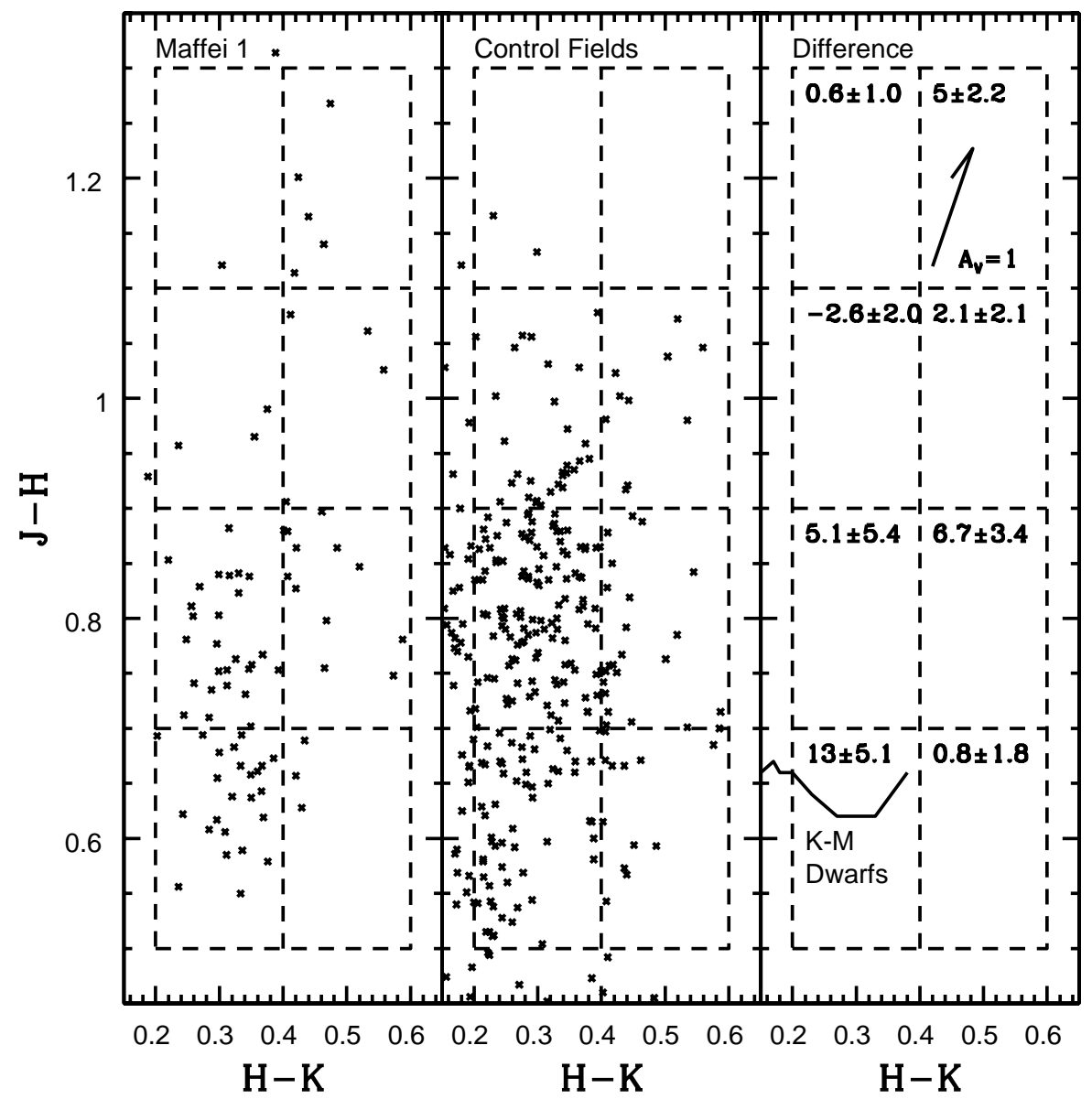

Fig. 8. - The left hand panel shows the $(J-H, H-K)$ TCD of objects with $K$ between 16.6 and 18.0 that are located between 20 and 90 arcsec from the center of Maffei 1 in Pointings 2, 3, and 4, while the middle panel shows the TCD of objects with $K$ between 16.6 and 18.0 in both control fields. The dashed lines in each panel define the $0.2 \times 0.2$ magnitude cells that are used to investigate the distribution of objects on the TCD. The entries in the right hand panel show the difference between the number of objects in the corresponding cell in the left hand panel and the number in the middle panel, with the latter scaled to the area in Maffei 1 that was searched for clusters. An excess population of sources is present at roughly the $2-\sigma$ significance level in three cells that span a range of $H-K$ and $J-H$ colors. A reddening vector with a length corresponding to $\mathrm{A}_{V}=1$ magnitude is also shown in the right hand panel, as is the sequence for solar neighborhood $\mathrm{K}$ - M main sequence stars from Bessell \& Brett (1988). 


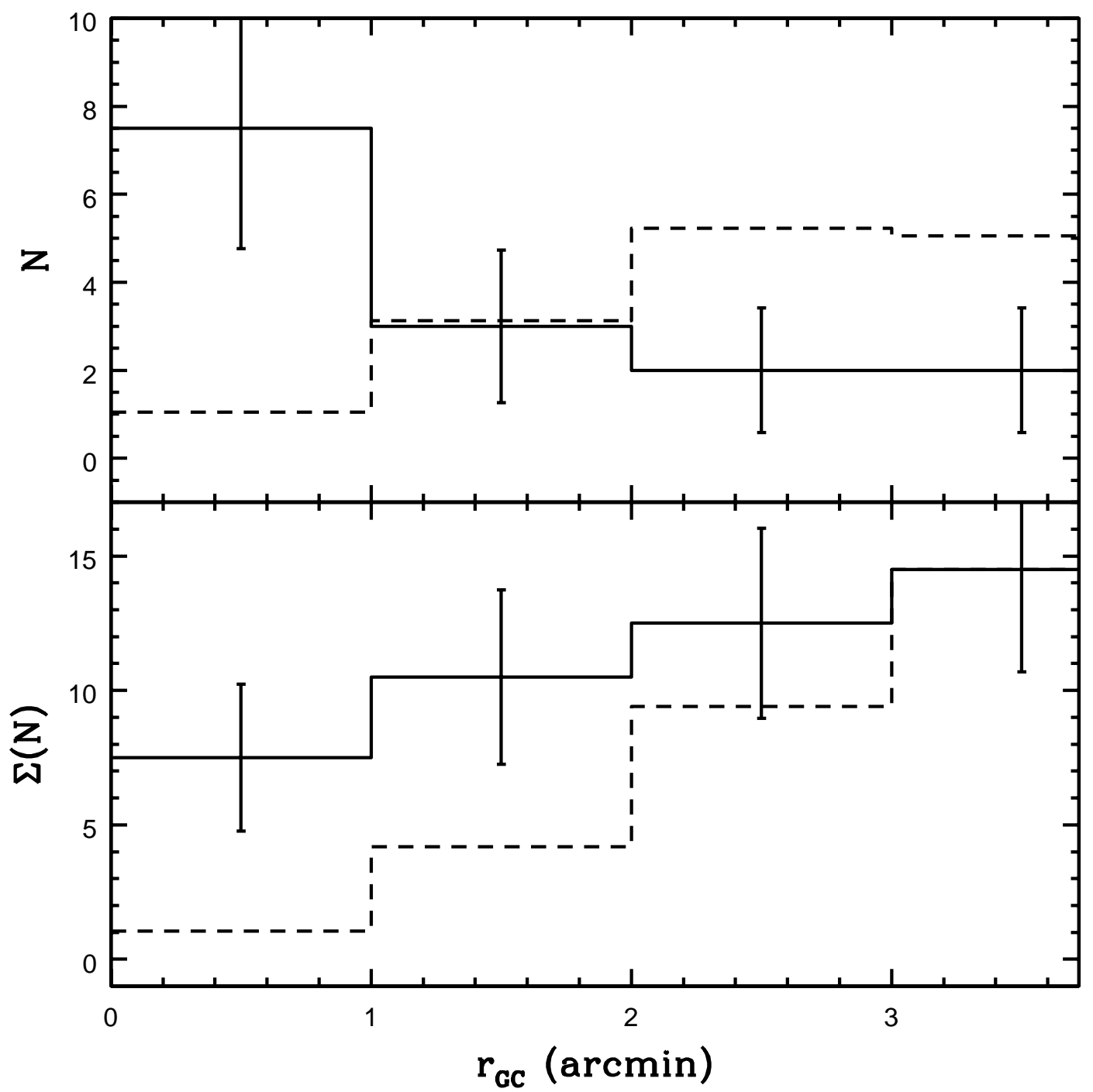

Fig. 9.- The radial distribution of very red objects detected in Maffei 1. The solid line in the top panel shows the number of cluster candidates with $H-K$ between 0.4 and 0.6 , and $J-H$ between 1.1 and 1.3 in 1 arcmin wide radial bins, centered on the nucleus of Maffei 1. The dashed line shows the number of objects predicted per radial interval assuming that they are uniformly distributed. The solid line in the lower panel shows the cumulative number of cluster candidates in the same four radial bins, summing outwards to larger radii, while the dashed line again shows the trend for a uniformly distributed population. It is evident that the red objects are not uniformly distributed, but are concentrated near the center of Maffei 1 , as expected if they are globular clusters. 


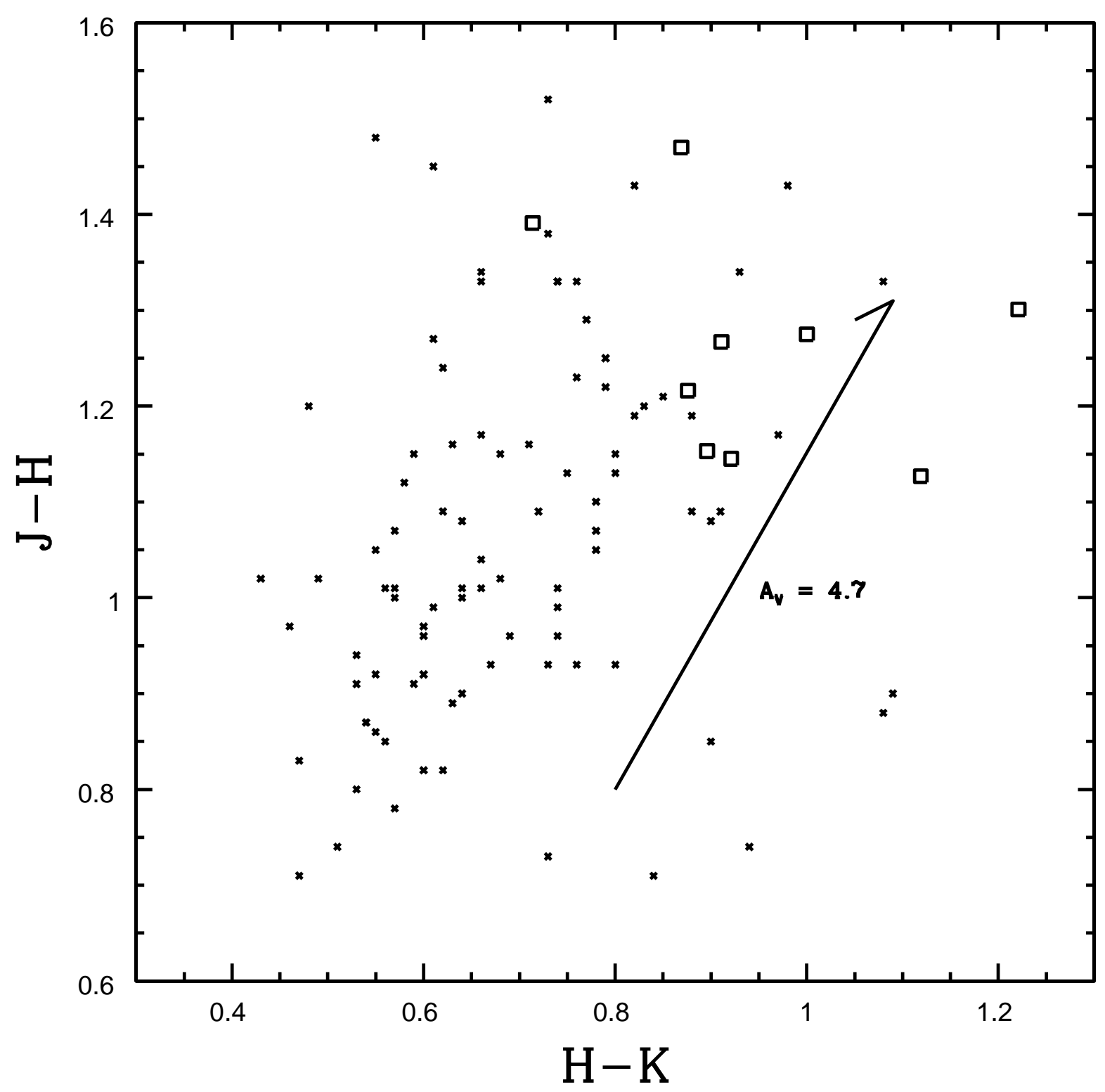

Fig. 10.- The $(J-H, H-K)$ TCD of spectroscopically confirmed L dwarfs (crosses) from Kirkpatrick et al. $(1999,2000)$ and the very red objects detected in our CFHTIR data (open squares). The arrow is the reddening vector, with an amplitude corresponding to $\mathrm{A}_{V}=4.7$. Note that the very red objects in the CFHTIR data have near-infrared SEDs that are similar to the majority of L dwarfs in the Kirkpatrick et al. (1999, 2000) sample. 